\title{
Satisfacción laboral de los emprendedores en México, retos y beneficios
}

\section{Job satisfaction of entrepreneurs in Mexico, challenges and benefits}

\author{
Humberto Charles-Leija*1, Jonathan Aguirre Peña ${ }^{2}$, \\ Rogelio Sánchez Rodríguez ${ }^{2}$ \\ ${ }^{1}$ Universidad Tecmilenio, Instituto de Ciencias de la Felicidad y Tecnológico de Monterrey, \\ Escuela de Negocios, México \\ ${ }^{2}$ Tecnológico Nacional de México, Instituto Tecnológico de Saltillo, México
}

Recibido el 22 de abril de 2019; aceptado el 25 de junio de 2020

Disponible en Internet el: 2 de septiembre de 2020

\section{Resumen}

El objetivo de la investigación es contrastar los niveles de satisfacción laboral entre emprendedores y empleados. Para el estudio se usan datos de la Encuesta de Bienestar Autorreportado (Biare) del 2014. Se estima un modelo logit ordenado utilizando como variable dependiente el nivel de satisfacción laboral. El estudio identificó que aquellas personas que emprenden tienen $4.07 \%$ más probabilidades de declararse muy satisfecho con su situación laboral. La originalidad del trabajo radica en ser pionero en el estudio de los emprendedores en referencia a su bienestar subjetivo en México.

Código JEL: I31, J24, L26

Palabras clave: Pymes; Bienestar subjetivo; Satisfacción laboral; Emprendedor

\footnotetext{
*Autor para correspondencia. Correo electrónico:humbertocharles@yahoo.com (H. Charles-Leija).

La revisión por pares es responsabilidad de la Universidad Nacional Autónoma de México.
} 


\begin{abstract}
The objective of the research is to compare the levels of job satisfaction between entrepreneurs and employees. For the study, data from the Self-Reported Welfare Survey (Biare) of 2014 are used. An ordered logit model is estimated using as a dependent variable the level of job satisfaction. The study identified that entrepreneurs are $4.07 \%$ more likely to declare themselves very satisfied with their employment situation. The originality of the work lies in being a pioneer in the study of entrepreneurs and their subjective well-being in Mexico.
\end{abstract}

JEL Code: I31, J24, L26

Keywords: SMEs; Subjective well-being; Job satisfaction; Entrepreneurship

\title{
Introduction
}

El emprendedor es un generador de crecimiento económico, crea una empresa, asume los riesgos financieros y dedica tiempo y esfuerzo a la misma (Freire Seoane y Teijeiro Álvarez, 2009). La actividad emprendedora es clave para la creación de empleo, competitividad e innovación (Moriano León, Palací Descals, y Morales Domínguez, 2006). Las nuevas empresas permiten una mayor competencia en el mercado de bienes, generan empleo y contribuyen al crecimiento de los países. A partir de lo anterior es que los gobiernos promueven la generación de nuevos emprendimientos como un aspecto clave de su agenda de desarrollo (Block y Koellinger, 2009).

El presente estudio parte de un marco teórico de bienestar subjetivo, es decir, se trabaja con auto reporte de los individuos. La mayor parte de los estudios de bienestar subjetivo en el trabajo han centrado el análisis en el trabajador por cuenta ajena (Freeman, 1978; Ohtake, 2012; Winkelmann y Winkelmann, 1998). Sin embargo, la figura del emprendedor ha cobrado relevancia en años recientes (Binder y Coad, 2013; Bradley y Roberts, 2004), pero, la mayoría de los estudios se han llevado a cabo en Estados Unidos y Europa (Blanchflower y Oswald, 1998; Block y Koellinger, 2009; Carree y Verheul, 2012).

Las investigaciones de bienestar subjetivo permiten enriquecer a campos como la administración, el desarrollo organizacional, la mercadotecnia y la economía (Clark y Oswald, 1994; Naudé, Amorós, y Cristi, 2014). Cada vez hay más estudios enfocados en el bienestar subjetivo en México (Charles-Leija, Aboites, y Llamas, 2018; Temkin, 2016). Algunos han vinculado el tema del emprendimiento y bienestar subjetivo o felicidad (Charles-Leija, Sánchez Rodríguez, Ramírez Jaramillo, y Aguirre Peña, 2019; Naudé et al., 2014).

El objetivo del presente estudio es identificar si existe mayor satisfacción laboral-personal por ser emprendedor respecto a tener un trabajo por cuenta ajena. Conviene precisar que los 
datos no permiten integrar una distinción entre la satisfacción laboral personal y la satisfacción laboral-profesional. La investigación se divide en cinco secciones, la primera es introductoria; la segunda aborda los antecedentes teóricos y empíricos relacionados con el bienestar subjetivo de los emprendedores; la tercera describe los datos y la metodología empleada en la investigación; la cuarta presenta los resultados; finalmente se muestran conclusiones.

\section{Antecedentes teóricos y empíricos}

Los emprendedores son los trabajadores que cuentan con mayor posibilidad de hacer adaptaciones a sus procesos para lograr una mejora en la experiencia (Devotto y Wechsler, 2019; Yepes-Baldó, Romeo, Westerberg, y Nordin, 2018). El emprendimiento puede ser particularmente positivo para individuos extrovertidos, simpáticos y diligentes (Berglund, Johansson Sevä, \& Strandh, 2016). Los emprendedores tienen motivaciones intrínsecas como aprovechar sus habilidades, generar empleo en la comunidad, así como extrínsecas, como los beneficios puramente pecuniarios (Deci y Ryan, 2000). Cuando el emprendimiento tiene un sentido intrínseco se puede asociar a los aspectos eudaimónicos y virtuosos que señalaba Aristóteles (349AD). A continuación, se describen los principales retos y satisfacciones relacionados con la actividad de emprender, que pueden tener un impacto significativo en la satisfacción laboral.

\section{Retos para los emprendedores}

Emprender es un trabajo muy exigente. Ser propietario de una pyme representa largas jornadas laborales y cargas de trabajo pesadas y estresantes para los propietarios. Emprender puede ser una actividad muy demandante y perjudicial para la salud física y psicológica, la carga de trabajo puede ser vista como un reto o como una amenaza por el emprendedor (Patel, Wolfe, y Williams, 2019). Así, la incertidumbre e inestabilidad laboral junto a la posibilidad de pérdidas potenciales de ingreso son elementos perjudiciales para la salud del emprendedor (Patel et al., 2019). Los aspectos anteriores son vividos en menor intensidad por aquellas personas que trabajan por cuenta ajena. En las primeras etapas de una pyme los emprendedores pueden enfrentarse a elementos que no habían considerado, los cuales pueden llevarles a procesos de frustración por la inestabilidad laboral y el costo de oportunidad manifestado en el tiempo laboral remunerado que están perdiendo, aunado a la inversión en capital.

Un aspecto negativo del emprendimiento es la posibilidad de que el emprendedor se explote a sí mismo. Han (2014) plantea que en el capitalismo del siglo XXI la explotación no proviene necesariamente de un patrón, sino que cada individuo puede, voluntariamente, ejercer una presión excesiva sobre sí mismo. Esta situación puede ser todavía más intensa en 
el contexto de los emprendedores, quienes se ven forzados a superar retos administrativos constantes a cambio de mantener su organización a flote.

Los problemas para financiarse son el desafío primordial de la mayoría de los emprendedores. Estudios previos han identificado que los emprendedores no conocen las opciones de financiamiento disponibles (De León, García Pimentel, y Ramírez Jaramillo, 2018) y, por tener un control inadecuado de los procesos cuentan con menores probabilidades de ser susceptibles de apoyo de parte de las micro financieras (Raccanello y Saucedo Carranza, 2015).

Se ha documentado que a partir del arranque de la "guerra contra el narcotráfico" a finales de 2006, en México crecieron distintos tipos de violencia, tales como asesinatos, secuestros y extorsiones. Empresarios y emprendedores se enfrentaron en mayor medida a un tipo de extorsión conocido como “derecho de piso” (Muñiz y Ramírez, 2015). Donde la víctima debe realizar pagos periódicos a delincuentes a cambio de no sufrir ataques. La violencia asociada al crimen organizado en México fue causante de la migración de más de 250 mil personas en los primeros cinco años de la guerra contra el narcotráfico (Ríos, 2012). La extorsión a microempresarios se volvió para las organizaciones delictivas una manera de obtener flujos rápidos de efectivo.

Un emprendedor pone en riesgo su estabilidad financiera en su empresa. Mientras un trabajador formal recibe beneficios de pertenecer a una empresa establecida, ingresos monetarios periódicos como aguinaldo, vales de despensa, etc., un emprendedor carece de ellos. Las dos prestaciones, cuya ausencia podrían afectar más a los emprendedores son la salud y el ahorro para el retiro. Un emprendedor tiene sesgos de exceso de confianza y normalmente no planea de manera adecuada su retiro. Asimismo, se le dificulta la obtención de derechos sociales como el seguro médico.

En México se ha documentado que el ahorro para el retiro previsto por su población es inadecuado e insuficiente, en parte porque los trabajadores están desinformados y desinteresados al respecto (Villagómez, 2014). El beneficio del ahorro para el retiro, con el que cuentan los trabajadores asalariados mediante mecanismos formales, surge a partir de un "ahorro forzado" por el estado. Los trabajadores por cuenta propia no son forzados a lo mismo y no planifican correctamente los esquemas que seguirán para lograr un retiro con ingresos suficientes para el sostenimiento propio y de la familia (Villagómez, 2014)

Se ha identificado una correlación negativa entre el promedio de felicidad de los países y la tasa de personas que poseen su propia empresa (Naudé et al., 2014). Lo anterior podría sugerir que una mayor proporción de pequeños negocios no necesariamente genera mayor bienestar subjetivo en los individuos. Los autores proponen que algunos propietarios de pequeñas empresas no asumieron ese rol por elección propia, sino que las circunstancias los orientaron. Una causa puede ser tener bajo capital humano y con ello menores posibilidades de obtener un ingreso elevado como persona asalariada o enfrentarse a una situación de desempleo. 


\section{Satisfacción laboral y emprendimiento}

Una pregunta que puede surgir de inicio es si existe una relación entre la satisfacción laboral y el emprendimiento. El emprendedor está más involucrado y comprometido con la empresa que el empleado regular. Para el empleado existe un componente de incertidumbre en el sentido de que la organización para la cual labora puede seguir, aunque él no esté. Para la mayoría de los emprendedores no ocurre la misma situación. En el caso de los emprendedores, se da con frecuencia que ellos mismos "son" la empresa. El funcionamiento de la organización depende del nivel de esfuerzo del emprendedor.

Emprender genera satisfacciones. Aquella persona que arranca una organización puede aplicar en ella sus conocimientos, habilidades y competencias. Emprender origina en las personas un sentimiento de autonomía clave para la satisfacción personal, razón que podría explicar que un emprendedor tiene menores tasas de ausentismo, mayor puntualidad, mejor desempeño, así como mayor compromiso organizacional en general (Bradley y Roberts, 2004). El emprendimiento puede verse como un mecanismo que disminuye las dificultades asociadas al problema del agente y el principal. Entre los elementos que más pueden contribuir a la satisfacción generada por los emprendedores por desempeñarse como autónomos puede ser: la autonomía, la flexibilidad y el trabajo por sí mismo (Block y Koellinger, 2009).

Emprender puede generar reconocimiento social. En países como Estados Unidos, el emprendedor es un personaje admirado (Bradley y Roberts, 2004). Se le puede asociar a valores muy respetados por el norteamericano, los vinculados a la movilidad social a través de la fundación de una propia empresa. En Europa los emprendedores son vistos como los "héroes" de la economía global (Berglund, Johansson Sevä, y Strandh, 2016). Emprender permite generar empleos y bienestar para la comunidad. Lo cual genera un estatus positivo para el emprendedor.

Emprender lleva a estados de fluir. Para los emprendedores puede que lo más importante no sea necesariamente el ingreso obtenido por su actividad en el mercado de trabajo. Se puede considerar que ellos no disfrutan solamente el resultado, sino también el proceso (Benz y Frey, 2008; Block y Koellinger, 2009). En psicología a ese proceso le han llamado estado de "flow" (Csikszentmihalyi, 2008). Diversos estudios han identificado mayor satisfacción laboral de los emprendedores respecto de los empleados regulares (Berglund et al., 2016).

Los emprendedores regularmente tienen ingresos menores que los trabajadores por cuenta ajena (Block y Koellinger, 2009). Si bien la economía tradicional marca al ingreso como uno de los principales incentivos de los individuos, la economía de la felicidad proponer que existen muchos dominios de vida importantes para las decisiones que toman las personas (Rojas, 2007). El bienestar de los individuos no solamente depende de los ingresos, sino de muchos 
componentes como el plano afectivo y emocional (Rojas, 2015). La intención de emprender tiene motivaciones más allá de los aspectos pecuniarios (Koellinger, Minniti, y Schade, 2007).

Las mujeres, en términos de emprendimiento, enfrentan problemas de restricciones de tiempo. Socialmente es esperado que ellas encaminen sus esfuerzos al trabajo doméstico. Se ha señalado que las mujeres que emprenden suelen sentir que al dedicar tiempo a su emprendimiento descuidan a su familia y sus responsabilidades de hogar (Moriano León et al., 2006), de la misma manera se ha documentado una mayor propensión por emprender de parte de los hombres (Koellinger et al., 2007). Otro dato relevante es que el promedio de mujeres que cuenta con contrato firmado en su empleo es menor que el de los hombres (30 contra 44\%, respectivamente) (Hernández García y Tomé González, 2016). Lo cual es un indicador de inseguridad laboral para las trabajadoras.

Emprender en lugar de trabajar representa un costo de oportunidad para las personas con mayor nivel educativo (Escamilla Salazar, Caldera González, y Cruz del Castillo, 2014)el emprendimiento ha adquirido gran importancia por su relación con el crecimiento y desarrollo económico de un país a través de la creación de empresas. En este sentido, el descubrimiento de oportunidades resulta un aspecto clave en la actividad empresarial y con ello convirtiéndose en un punto muy importante en las investigaciones (las cuales han mejorado con datos del Global Entrepreneurship Monitor (GEM. Dado lo anterior se puede esperar que aquellas personas con maestría o doctorado se sentirán más satisfechas si se desempeñan en una actividad por cuenta ajena. Las personas con mayor capital humano regularmente están muy especializadas en un tema y presentan dificultad para llevar a cabo la serie de responsabilidades diferentes que tiene un emprendedor. Cabe resaltar que la formación profesional es relevante para lograr un buen desempeño en una empresa propia. Contar con una licenciatura debe crecer las posibilidades del éxito de una microempresa. Principalmente si el emprendedor se forma en aspectos como administración, contabilidad, mercadotecnia, liderazgo, etc. El emprendimiento presenta una correlación negativa con el nivel educativo, salvo algunos países ricos en donde se ha observado significancia positiva entre posgraduados (Koellinger et al., 2007).

Por otra parte, el grado de licenciatura puede contribuir al éxito de los emprendimientos (Davidsson y Honig, 2003). Las personas con mayor preparación académica pueden formar mejores planes estratégicos en las empresas. Si no se cuenta con suficiente preparación académica el emprendimiento puede ser un factor generador de estrés. Estudios previos han señalado que para incrementar las posibilidades de éxito de un negocio es crucial echar mano de un plan de negocios (Charles-Leija, Sánchez, y Ramírez Jaramillo, 2020). Para que el emprendimiento puede generar bienestar en los propietarios, el emprendedor debe percibir que existen altas posibilidades de éxito. La escolaridad no solamente es un medio que permite crecer la productividad y el comportamiento cívico de los individuos, también permite definir las relaciones laborales (Varela Llamas, Ocegueda Hernández, Castillo Ponce, 
y Huber Bernal, 2016). Dado lo anterior es posible suponer que las personas que cuentan con licenciatura pueden lograr un mayor reconocimiento de sus empleados a la hora de emprender.

Estudios previos han apuntado a que los jóvenes universitarios son más proclives a querer trabajar por cuenta ajena que a volverse emprendedores (Moriano León et al., 2006). Por otro lado, se ha identificado que las personas en edad avanzada que arrancan una nueva empresa reportan niveles más elevados de calidad de vida (Kautonen, Kibler y Minniti, 2017). Tal situación puede ocurrir porque a través del emprendimiento los jubilados pueden mantenerse activos, aprovechar sus conocimientos desarrollados a través de los años siendo empleados y aprovechar el capital financiero que pudieron haber logrado. De esta manera el emprendedor de edad adulta no enfrenta las restricciones de capital y experiencia que si enfrentan los emprendedores jóvenes.

En cuanto a tamaño de la empresa un elemento que se puede esperar es que la satisfacción laboral crezca con el tamaño de la organización. Una empresa de gran tamaño se beneficia con elementos como la economía de escala, lo cual permite mejores salarios y estabilidad para sus empleados. Lo anterior difícilmente ocurre en el contexto de los emprendedores, sobre todo si son novatos. Quien trabaja por su propia cuenta encuentra complicado desarrollar en una generación una empresa de gran tamaño. Para los emprendedores la sola supervivencia de la pyme es un reto mayúsculo. Se espera que el emprendedor obtenga un beneficio personal subjetivo a partir de conseguir que su empresa nazca, crezca y se desarrolle. Para un emprendedor la satisfacción puede provenir de integrar la "aventura" de emprender (Wiklund, Nikolaev, Shir, Foo, y Bradley, 2019).

La satisfacción del emprendedor puede provenir, como ya han señalado diversos autores: de la autonomía de ejercer una actividad gratificante, de la oportunidad de aprovechar habilidades y conocimientos en un establecimiento. Otro aspecto crucial puede ser que el emprendedor está salvando una situación de subempleo. Problema que es cada vez más creciente en México (Yamada y Oviedo, 2016). A continuación, se presenta un esquema que describe las variables de interés en el presente estudio. 


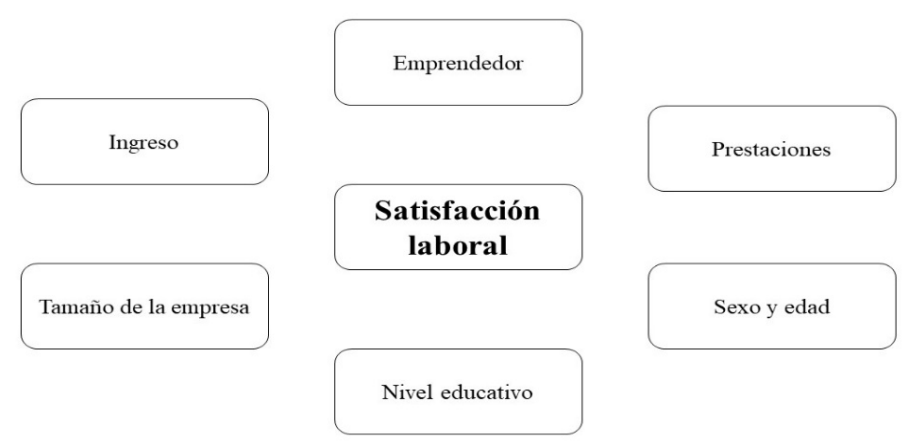

Figura 1.Variables que inciden en la satisfacción laboral

Fuente: Elaboración propia.

\section{Datos y metodología}

\section{Datos}

En esta investigación se entiende "emprendedor" al encuestado que manifiesta ser trabajador independiente. Mientras el "empleado por cuenta ajena" es quien señaló ser trabajador subordinado. Para el estudio se utilizó la Encuesta de Bienestar Autorreportado (BIARE) llevada a cabo por el Instituto Nacional de Estadística y Geografía (INEGI, 2014), dentro de la Encuesta de Ingreso y Gasto de los Hogares (ENIGH). La muestra, representativa para México, es de 24,440 trabajadores, de los cuales 18,325 son subordinados y 6.1155 son emprendedores. Estudios previos han empleado la ENIGH para estimar aspectos laborales como ingresos por salario (Varela Llamas et al., 2016) y búsqueda de empleo (Charles-Leija, Torres, y Castro, 2018).

En la tabla 1 se observan los valores promedios de las variables de interés del estudio, se observa que en el mercado laboral existen un porcentaje mayor de hombres que de mujeres, siendo ligeramente mayor la diferencia en el campo del emprendimiento. Otra diferencia de interés es la edad entre los dos grupos, se identifica un promedio de edad mayor en los trabajadores por cuenta propia, tema que puede relacionarse con el hecho de que este grupo de población sigue trabajando incluso a edades avanzadas. Relacionado con la edad está el estado conyugal, donde los porcentajes de emprendedores casados son mayores, respecto de los solteros. 
Estadística descriptiva de variables demográficas del estudio

\begin{tabular}{lcc}
\hline & Emprendedor & Empleado \\
\hline Variable & Promedio & Promedio \\
Mujer & 0.45 & 0.41 \\
Edad & 47.78 & 37.78 \\
Soltero & 0.12 & 0.24 \\
Casado & 0.52 & 0.42 \\
Separado & 0.09 & 0.09 \\
Divorciado & 0.03 & 0.03 \\
Viudo & 0.08 & 0.03 \\
Unión libre & 0.17 & 0.19 \\
$\mathrm{~N}$ & 6,115 & 18,325 \\
\hline
\end{tabular}

Fuente: Elaboración propia con datos de BIARE 2014.

El ítem central del estudio, que se emplea como variable independiente es "¿Qué tan satisfecho está con su actividad principal?". El rango de respuestas que los individuos pueden dar va de cero a 10. En este sentido, ser empleado por cuenta ajena permite al trabajador lograr una mayor especialización en una operación específica. De esta manera, quien enfoca sus esfuerzos exclusivamente en su desarrollo profesional como subordinado tiene más posibilidades de obtener un ingreso elevado por sus competencias como empleado. Lo anterior apunta a que a las personas con mayor nivel educativo les puede convenir más la condición de subordinado ya que aprovechan mejor su especialización.

Un aspecto relevante del emprendedor en México es la dificultad que enfrenta al momento de querer jubilarse. Para un trabajador que labora por cuenta ajena y cuenta con prestaciones existe la posibilidad de una pensión al finalizar su etapa laboral. Para los microempresarios esa posibilidad es casi nula. La figura 2 muestra los histogramas de poblaciones de emprendedores y subordinados a partir de su edad, en ella se observa que los emprendedores no tienen el hábito del ahorro lo suficientemente arraigado y eso dificulta la posibilidad de retirarse del mercado laboral a una edad semejante a la que lo hacen los trabajadores por cuenta ajena. 


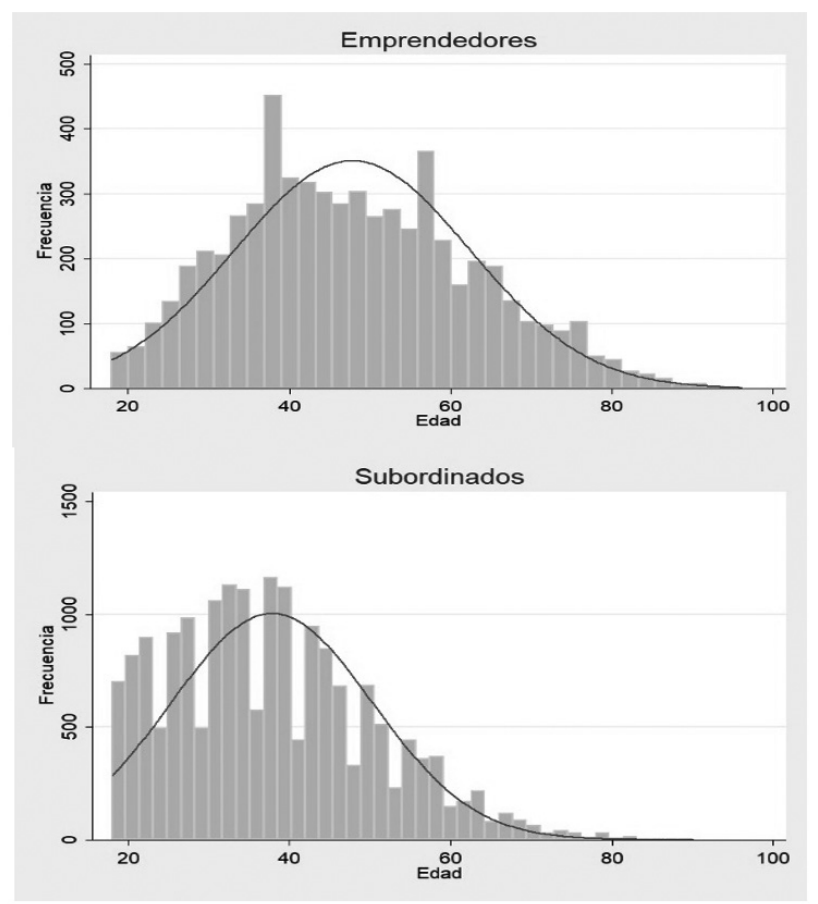

Figura 2 .Frecuencia de edades de trabajo, emprendedores y subordinados, México 2014

Fuente: Elaboración propia con datos de BIARE 2014.

Si bien, tanto emprendedores como trabajadores por cuenta ajena tienen un promedio de edad de 37 años, la figura 2 muestra que aquellas personas que cuentan con su propio establecimiento siguen trabajando incluso habiendo rebasado los 60 años de edad. La necesidad de seguir siendo económicamente activo juega en perjuicio de varios aspectos de la calidad de vida de las personas. Así, se observa que una característica importante del empleo subordinado es que puede ofrecer la posibilidad de una jubilación y un retiro del mercado laboral a una edad avanzada. Situación que es más complicada de llevar a cabo por parte de los emprendedores. Otro aspecto a señalar es la cantidad de prestaciones con las que cuentan los emprendedores y los empleados. Los emprendedores encuestados cuentan, en promedio, con 0.03 prestaciones, mientras los trabajadores por cuenta ajena manifiestan 4.8 prestaciones $^{1}$.

\footnotetext{
${ }^{1}$ Las prestaciones consideradas en la encuesta son 19: incapacidad, aguinaldo, vacaciones, reparto de utilidades, crédito de vivienda, guarderías, cuidados maternos, SAR o AFORE, seguro de vida, préstamos, prima vacional,
} 


\section{H. Charles-Leija, et al./ Contaduría y Administración 66(3) 2021, 1-20 \\ http://dx.doi.org/10.22201/fca.24488410e.2021.2524}

La tabla 2 presenta el nivel educativo promedio de emprendedores y trabajadores asalariados. En ella se observa que cerca del $47 \%$ de las personas que están a cargo de una pyme no han logrado concluir la secundaria. Ello representaría que un porcentaje elevado de los emprendedores no tienen suficientes competencias técnicas ni habilidades gerenciales para lograr que sus establecimientos prosperen. Se observa que un segmento importante de personas que no cuentan con educación se dedican a trabajar por cuenta ajena. Esto se explica en cuanto a que su nivel de calificación les limita la posibilidad de un ingreso significativo siendo empleados de una organización.

Tabla 2

Frecuencia y porcentaje de los distintos niveles educativos para empleados y subordinados, México 2014

\begin{tabular}{lcccc}
\hline & \multicolumn{2}{c}{ Subordinado } & \multicolumn{2}{c}{ Emprendedor } \\
\hline Nivel educativo concluido & Frecuencia & Porcentaje & Frecuencia & Porcentaje \\
\hline Ninguno & 488 & 2.66 & 531 & 8.68 \\
Preescolar & 12 & 0.07 & 14 & 0.23 \\
Primaria & 3,891 & 21.23 & 2,362 & 38.63 \\
Secundaria & 5,248 & 28.64 & 1,491 & 24.38 \\
Preparatoria & 3,622 & 19.77 & 739 & 12.09 \\
Normal & 220 & 1.2 & 31 & 0.51 \\
Carrera técnica o comercial & 883 & 4.82 & 269 & 4.4 \\
Profesional & 3,534 & 19.29 & 613 & 10.02 \\
Maestría & 349 & 1.9 & 51 & 0.83 \\
Doctorado & 78 & 0.43 & 14 & 0.23 \\
\hline
\end{tabular}

Fuente: Elaboración propia con base en la BIARE, 2014.

En la tabla 3 se observa que las pymes tienen mayor presencia en entornos rurales. Siendo que el 52\% de los emprendedores habitan localidades con poblaciones menores a los 15 mil habitantes, mientras que únicamente el $33 \%$ de los subordinados habita en localidades de esa cantidad de habitantes.

apoyos educativos, servicio de comedor, crédito fonacot, ayuda de despensas, ayuda en el pago de servicios, pensión en caso de incapacidad permanente, pensión en caso de fallecimiento, otras. 
Tabla 3

Frecuencia y porcentaje de los distintos tamaños de localidad para empleados y subordinados, México 2014

\begin{tabular}{lcccc}
\hline & \multicolumn{2}{c}{ Subordinado } & \multicolumn{2}{c}{ Emprendedor } \\
\hline Tamaño de localidad & Frecuencia & Porcentaje & Frecuencia & Porcentaje \\
\hline Más de 100 mil hab. & 9,275 & 50.61 & 2,001 & 32.72 \\
Entre 15 y 99.999 mil hab. & 2,934 & 16.01 & 910 & 14.88 \\
Entre 2.5 y 14.999 mil hab. & 2,797 & 15.26 & 1,074 & 17.56 \\
Menos de 2.5 mil hab. & 3,319 & 18.11 & 2,130 & 34.83 \\
\hline
\end{tabular}

Fuente: Elaboración propia con base en la BIARE, 2014.

\section{Metodología e hipótesis}

Si bien la pregunta original hecha por INEGI en referencia al nivel de satisfacción establece que los individuos elijan un valor entre cero y 10. Para simplificar el análisis en el presente estudio se agruparon distintas respuestas para formular cuatro categorías de satisfacción entre las personas. Aquellos que respondieron entre cero y cuatro se consideraron personas "insatisfechas" con su trabajo, quienes respondieron datos entre cinco y seis se clasificaron como "poco satisfechos", los que contestaron entre siete y ocho pasaron a la categoría de "satisfechos", finalmente, los que contestaron nueve o diez, integran la categoría "muy satisfechos". En la investigación nos centraremos en los elementos que incidan en que los trabajadores (tanto emprendedores como subordinados) integren el nivel más elevado de satisfacción. Es importante resaltar que una limitante del estudio es la encuesta utilizada. Debido a aspectos presupuestales no fue posible el levantamiento de información de primera mano con representatividad nacional. Para el presente estudio se estima un modelo ordinal, puesto que la variable dependiente tiene establecida una jerarquía sobre las respuestas (Cameron y Trivedi, 2005). Los modelos ordenados consideran una variable latente

$$
y_{i}^{*}=x_{i}^{\prime} \beta+u_{i}
$$

$y i^{*}$ se incrementa a partir de umbrales desconocidos según el ordenamiento de las alternativas. Se puede definir como sigue:

$$
y_{i}=j \text { si } \alpha_{j-1}<y_{i}^{*} \leq \alpha_{j}
$$


Para un modelo con $m$ alternativas $\quad \alpha_{0}=-\infty$ y $\alpha_{m}=\infty$, entonces

$$
\begin{gathered}
\operatorname{Pr}\left[y_{i}\right]=\operatorname{Pr}\left[\alpha_{j-1}<y_{i}{ }^{*}<\alpha_{j-1}\right] \\
=\operatorname{Pr}\left[\alpha_{j-1}<x_{i}^{\prime} \beta+u_{i}<\alpha_{j-1}\right] \\
=\operatorname{Pr}\left[\alpha_{j-1}-x_{i}^{\prime} \beta<u_{i}<\alpha_{j-1}-x_{i}^{\prime} \beta\right] \\
=F\left(\alpha_{j-1}-x_{i}^{\prime} \beta\right)-F\left(\alpha_{j-1}-x_{i}^{\prime} \beta\right)
\end{gathered}
$$

$F$ es una función de distribución acumulativa (cdf) de $u_{i}$. Los $\beta$ son obtenidos a través de máxima verosimilitud y su signo identifica la dirección del impacto. Para cuantificar los efectos marginales se emplea la siguiente ecuación:

$$
\frac{\partial \operatorname{Pr}\left[y_{i}=j\right]}{\partial x_{i}}=\left\{F^{\prime}\left(\alpha_{j-1}-x_{i}^{\prime} \beta\right)-F^{\prime}\left(\alpha_{j}-x_{i}^{\prime} \boldsymbol{\beta}\right)\right\} \boldsymbol{\beta}
$$

La quinta ecuación se contrasta empleando un modelo logit ordenado (ologit) debido a que ofrece ventajas al momento de interpretar los resultados.

Satisfacción laboral $=f$ (emprendedor,variables de control)

Como variables de control para las regresiones de satisfacción laboral se emplearon: situación de emprendedor o subordinado, situación conyugal (d_soltero,d_ulibre, d_casado, d_separado y d_divoricado), nivel educativo (d_hasta_sec, d_prof y d_posg), sexo, tamaño de la empresa (tam_emp), ingresos como emprendedor y como asalariado (1_ingtrab), edad y edad al cuadrado (edad2), sector de actividad económica y total de prestaciones laborales (t_pres). La hipótesis del estudio es que los emprendedores tendrán mayor nivel de satisfacción laboral que los empleados por cuenta ajena.

\section{Resultados}

En la tabla 4 se pueden observar los efectos marginales de la satisfacción laboral de emprendedores y subordinados. Cuando se toman en cuenta todos los trabajadores se puede apreciar que ser emprendedor representa un $4.07 \%$ mayor de posibilidades de declararse muy satisfecho con su situación laboral. Ello se puede explicar a partir de una percepción de realización personal, libertad para tomar decisiones dentro de la organización, etc. 
Como era de esperarse, un mayor ingreso, tanto siendo empleado por cuenta ajena como por cuenta propia tiene repercusiones positivas sobre la satisfacción laboral. Considerando a todos los trabajadores se aprecia que cada nuevo nivel escolar alcanzado representa mayor satisfacción laboral. En ese caso se tomó educación básica (nivel secundaria o menor) como variable de referencia para evitar multicolinealidad. Así, quien tiene posgrado cuenta con $9 \%$ más probabilidades de declararse muy satisfecho con el plano laboral que respecto de aquellos que cuentan únicamente con secundaria o primaria. Se observa que contar con prestaciones es un elemento estadísticamente significativo para quienes son empleados por cuenta ajena.

El estudio también muestra que no existe mayor satisfacción laboral estadísticamente significativa para quien cuenta con posgrado y emprende, este resultado puede relacionarse con que haber dedicado muchos años a la formación profesional implica un mayor costo de oportunidad para quien intenta ser su propio jefe. Ello apuntaría a que ser subordinado genera mayor satisfacción laboral entre las personas con maestría y/o doctorado. En el caso de los profesionistas, sí existe una prima en términos de satisfacción si se incorpora al mercado laboral desde el emprendimiento. En este sentido se confirma lo planteado por la teoría económica en referencia a que el costo de oportunidad de emprender impide un impacto positivo en la satisfacción laboral para los emprendedores con posgrado.

Los resultados de la tabla anterior destacan que el único nivel educativo que genera un efecto significativo en la satisfacción laboral de los emprendedores lo genera contar con una licenciatura. Tal situación es comprensible y a que poseer menor preparación limita las competencias administrativas de los emprendedores. De la misma manera tener mayor escolaridad repercute en un mayor costo de oportunidad por emprender.

Otro elemento para destacar es el tamaño de la empresa, si bien para un empleado por cuenta ajena esta variable no representa un cambio significativo en su bienestar, sí ocurre en el caso del emprendedor. Lo cual puede tener varias explicaciones. Una es que cada empleado nuevo representa la satisfacción personal de un empleo más generado. Cuando se genera un empleo nuevo se está contribuyendo de manera muy positiva a la sociedad. Un nuevo trabajador es un triunfo significativo para el emprendedor que empieza a lograr la transición a empresario. Desafortunadamente, en México, cuando una pyme empieza a crecer, sus empleados no necesariamente obtienen los beneficios que obtendrían en una empresa grande. Es frecuente que las empresas de menor tamaño no brinden a sus trabajadores las prestaciones mínimas requeridas por la ley. 
Tabla 4

Efectos marginales de la satisfacción laboral, México 2014

\begin{tabular}{lccc}
\hline Variable & $\begin{array}{c}\text { Todos los } \\
\text { trabajadores }\end{array}$ & Emprendedores & Subordinados \\
\hline emprendedor (d) & $0.047^{* * *}$ & & \\
sexo (d) & $0.026^{* * *}$ & -0.012 & $0.037 * * *$ \\
ln_ingtrab & $0.007^{* * *}$ & 0.003 & $0.017 * * *$ \\
edad & $0.003^{* *}$ & 0.002 & 0.002 \\
edad2 & 0.000 & 0.000 & 0.000 \\
d_ulibre (d) & 0.004 & -0.011 & 0.006 \\
d_casado (d) & $0.021^{*}$ & 0.012 & $0.021 *$ \\
d_separado (d) & $-0.041^{* *}$ & -0.043 & $-0.039 * *$ \\
d_divorciado (d) & -0.009 & 0.017 & -0.015 \\
d_bach (d) & $0.024 * *$ & 0.015 & $0.023 * *$ \\
d_prof (d) & $0.045^{* * *}$ & $0.084 * * *$ & $0.032^{* *}$ \\
d_posg (d) & $0.099^{* * *}$ & 0.096 & $0.089 * *$ \\
sector & $0.000^{*}$ & 0.000 & $0.001 * *$ \\
tam_emp & 0.000 & $0.020^{*}$ & 0.000 \\
t_prestac & $0.008^{* * *}$ & 0.000 & $0.008 * * *$ \\
\hline
\end{tabular}

Fuente: Elaboración propia con base en la BIARE, 2014.

Al distinguir por sexo se observa que no hay diferencia para el caso de los emprendedores, pero si para los empleados. El signo positivo asociado a la variable apunta a que existe mayor satisfacción laboral para los hombres respecto de las mujeres, entre aquellos que trabajan por cuenta ajena. Ello se puede asociar a las ventajas laborales que se presentan para los hombres respecto de las mujeres. Una de ellas el llamado "techo de cristal", el cual describe las barreras que enfrentan las mujeres para crecer de manera profesional (Silva, Dal Magro, Gorla, y Silva, 2018).

\section{Discusión}

Cuando un individuo emprende lo hace a partir de altas expectativas. Le interesa generar empleos, aprovechar sus habilidades, no recibir órdenes de un jefe. Cuando los individuos son exitosos en esas metas se sienten satisfechos. Sin embargo, existen muchos factores que pueden llevar al fracaso de una empresa: la falta de una organización adecuada, la inseguridad, los ciclos económicos negativos, etc.

A partir de 2006 la delincuencia diversificó sus negocios criminales y en México se volvió cada vez más frecuente la extorsión a empresarios y emprendedores (Ríos, 2012). Estudios sobre 
contenido noticioso de agresiones a empresarios y emprendedores mostró que los noticiarios mostraban una mayor cobertura de las agresiones contra los empresarios y emprendedores que contra su empresa (Muñiz y Ramírez, 2015). Este resultado muestra que quien emprende está más expuesto a noticias que refieren a los daños que puede recibir en su persona, lo cual permite crecer su miedo e insatisfacción referente a su situación.

Los emprendedores en México son, más que creadores de empleo, creadores de autoempleo. Distintas condiciones los limitan para lograr crecer sus organizaciones. Las tasas de muerte de las pymes mexicanas son elevadas y por su condición de informalidad dificultan ser una fuente de ingresos fiscales para el estado. Así, el emprendimiento-autoempleo ocasionalmente se vuelve una estrategia para evitar el desempleo en un contexto donde un alto porcentaje de la población en México no cuenta con ahorros para sobrellevar periodos sin una fuente de ingresos laboral.

En temas de ahorro para el retiro los emprendedores pueden ser víctimas de un desconocimiento financiero sobre su capacidad de ahorro para el retiro. La posibilidad de jubilarse está afectada por el nivel de ahorro para el retiro y por el nivel de riqueza alcanzado por los trabajadores (Villagómez, 2014). Considerando lo anterior, si un emprendedor no tuvo la oportunidad de ahorrar en previsión al futuro y no alcanzó un nivel de riqueza significativo se ve en la necesidad de mantenerse laborando para sobrevivir. En lo referente al ahorro para el retiro también podría reflexionarse en que los auto-empleados pueden querer seguir trabajando en su empresa debido al "aprecio" que tienen por ella. A la gratificación que tienen de mantener operando un establecimiento del que son propietarios.

Si bien algunos autores han apuntado el emprendimiento desde la visión lucrativa (Shane y Venkataraman, 2000), la perspectiva de economía de la felicidad es más completa. A partir del marco teórico del bienestar subjetivo se puede observar que quien emprende no lo hace solamente por el carácter pecuniario. Hay elementos de autorrealización, de estados de fluir y de gratificación intrínseca. Un elemento crucial para reconocer cuando el emprendimiento puede ser fuente de satisfacción es realizarlo por propósitos intrínsecos, es decir, buscando una gratificación con la actividad realizada. Cuando el emprendimiento se realiza principalmente como medio de búsqueda de altos ingresos es más complicado que el emprendedor se sienta satisfecho (Sherman, Randall, y Kauanui, 2016).

La baja calidad educativa de algunas universidades puede generar abundancia de personas con licenciatura que no necesariamente cuentan con las competencias requeridas (Yamada y Oviedo, 2016). Dichas personas reducen los salarios para profesionistas y generan en algunos profesionales la necesidad de emprender para superar un posible subempleo. De esta manera, un licenciado, que en el mercado laboral podría estar sobre educado, en su propia empresa puede desempeñar sus habilidades y conocimientos. Es en esas poblaciones donde puede ubicarse la mayor satisfacción por emprender. 


\section{Conclusiones}

El presente estudio centró el análisis en la comparación entre emprendedor y trabajador subordinado, puntualizando las diferencias en satisfacción laboral. La investigación partió de la pregunta ¿Existen mayor satisfacción laboral por ser emprendedor respecto a tener un trabajo por cuenta ajena? Los resultados apuntaron a que la condición de emprendedor genera un 4.07\% mayor probabilidad de declararse muy satisfecho con su actividad principal. Asimismo, se observó que, de los elementos evaluados, los dos que representan efectos positivos sobre la satisfacción de los emprendedores son contar con licenciatura y que la empresa tenga un tamaño mayor.

Un hallazgo relevante del estudio fue el aspecto referente a las prestaciones. Se pudo observar que, para los asalariados, contar con prestaciones es un aspecto que ejerce una contribución positiva y significativa con su satisfacción laboral. En este sentido se aprecia que las personas que deciden emprender son aquellas que otorgan menor relevancia a ese aspecto, ya que el $95 \%$ de los emprendedores no cuenta con prestaciones.

El estudio identificó también que las personas con posgrado reportan mayor satisfacción laboral siendo trabajadores por cuenta ajena. El resultado es comprensible a partir del principio del costo de oportunidad. Las personas con posgrado han realizado una elevada inversión de tiempo en un campo del conocimiento y aspiran a mayores salarios, respecto de aquellos con menor nivel académico. Así, en caso de encontrar un empleo que le ofrezca una remuneración acorde a su nivel educativo, los más capacitados, sienten mayor satisfacción desempeñándose por cuenta ajena. Aunado a lo anterior, los emprendedores en la mayoría de las ocasiones deben tener habilidades variadas para atender los requerimientos de la organización y las personas con mayor educación académica tiene una variedad de conocimientos menor y más enfocada en un solo aspecto del conocimiento.

Una limitante del trabajo es la encuesta utilizada. Debido a aspectos presupuestales no fue posible la aplicación de una encuesta propia con representatividad nacional como ofrece INEGI. A partir de lo anterior, los investigadores no tuvimos la oportunidad de proponer los ítems para el instrumento de evaluación de la satisfacción de los encuestados. Algunos aspectos a estudiar en análisis futuros son: la cantidad de emprendimientos (o empleos) previos de los encuestados. Así como la cantidad de amigos o familiares emprendedores. Lo cual puede ser una señal de mayor comprensión del fenómeno emprendedor y su relación con el bienestar subjetivo. Otra línea de trabajo futuro puede apuntar a indagar en el bienestar subjetivo del emprendedor antes de arrancar su empresa y darle seguimiento a través del tiempo. O centrar el análisis en las mujeres emprendedoras. Trabajos a futuro pueden centrarse en el tipo de empresa y sector como elemento mediador del bienestar de los emprendedores. Identificar qué elementos contienen las microempresas que permiten la mayor satisfacción 
para el emprendedor. Si bien el rol del autónomo es dirigir una organización y ello implica determinadas cargas de trabajo y estrés. Es posible que exista un tipo de empresa que brinda mayores satisfacciones a los emprendedores.

En términos de políticas públicas el estudio contribuye a la reflexión de la jubilación para los trabajadores por cuenta propia. En ocasiones los emprendedores, al iniciar su empresa, eligen la informalidad para lograr un menor pago de impuestos, sin embargo, eso puede tener distintos efectos en su nivel de prestaciones. Desde la política pública es crucial atender los problemas que viven los emprendedores en términos de acceso a servicios de salud y un adecuado sistema de pensiones. El problema puede volverse un tema grave de salud pública si no se atiende de la manera adecuada.

En un contexto empresarial, el presente artículo contribuye al campo de la administración en brindar pautas respecto de algunos aspectos claves de la productividad de los trabajadores. Aquellos individuos que manifiestan mayor satisfacción laboral son más productivos, presentan menores tasas de rotación y generan mejor clima organizacional. Elementos como las prestaciones sociales pueden ser componentes clave que orienten a los trabajadores por cuenta ajena a tener una mayor satisfacción laboral y mejor rendimiento.

\section{Referencias}

Aristóteles. (349AD). Ética a Nicómaco (M. Araujo \& J. Marías, eds.). Madrid, España: Pólitica, Estudios Políticos y Constitucionales.

Benz, M., \& Frey, B. S. (2008). Being independent is a great thing: Subjective evaluations of self-employment and hierarchy. Economica, 75(298), 362-383. https://doi.org/10.1111/j.1468-0335.2007.00594.x

Berglund, V., Johansson Sevä, I., \& Strandh, M. (2016). Subjective well-being and job satisfaction among self-employed and regular employees: does personality matter differently? Journal of Small Business and Entrepreneurship, 28(1), 55-73. https://doi.org/10.1080/08276331.2015.1115699

Binder, M., \& Coad, A. (2013). Life satisfaction and self-employment: A matching approach. Small Business Economics, 40(4), 1009-1033. https://doi.org/10.1007/s11187-011-9413-9

Blanchflower, D. G., \& Oswald, A. J. (1998). What Makes an Entrepreneur? Journal of Labor Economics, 16(1), 26-60. https://doi.org/10.1086/209881

Block, J., \& Koellinger, P. (2009). I can't get no satisfaction - Necessity entrepreneurship and procedural utility. Kyklos, 62(2), 191-209. https://doi.org/10.1111/j.1467-6435.2009.00431.x

Bradley, D. E., \& Roberts, J. A. (2004). Self-Employment and Job Satisfaction: Investigating the Role of Self-Efficacy, Depression, and Seniority. Journal of Small Business Management, 42(1), 37-58. https://doi.org/10.1111/ j.1540-627X.2004.00096.x

Cameron, A. C., \& Trivedi, P. K. (2005). Microeconometrics. Methods and applications. https://doi.org/10.1017/ CBO9781107415324.004

Carree, M. A., \& Verheul, I. (2012). What Makes Entrepreneurs Happy? Determinants of Satisfaction Among Founders. Journal of Happiness Studies, 13, 371-387. https://doi.org/10.1007/s10902-011-9269-3

Charles-Leija, H., Aboites, G., \& Llamas, I. (2018). Una revisión de aportaciones que contribuyeron al estudio de la utilidad y la felicidad en la economía. Análisis Económico, 33(84), 57-76. 
Charles-Leija, H., Sánchez, R., \& Ramírez Jaramillo, A. L. (2020). Formulación y evaluación de proyectos, una reflexión para las pymes agroindustriales de México. Ciencias Administrativas, 8(16).

Charles-Leija, H., Sánchez Rodríguez, R., Ramírez Jaramillo, A. L., \& Aguirre Peña, J. (2019). Entrepreneurs in México, an approach to their quality of life. Dimensión Empresarial, 17(3). https://doi.org/10.15665/dem. v17i3.1646

Charles-Leija, H., Torres, A., \& Castro, D. (2018). Efectos del Capital Social en el empleo en México. Economía Institucional.

Clark, A. E., \& Oswald, A. J. (1994). Unhappiness and unemployment. The Economic Journal, 104(424), 648-659. https://doi.org/10.2307/2234639

Csikszentmihalyi, M. (2008). Flow. In HarperCollins e-Books. https://doi.org/10.1017/CBO9781107415324.004

Davidsson, P., \& Honig, B. (2003). The role of social and human capital among nascent entrepreneurs. Journal of Business Venturing, 18(3), 301-331. https://doi.org/10.1016/S0883-9026(02)00097-6

De León, S., García Pimentel, V., \& Ramírez Jaramillo, A. L. (2018). Programas federales de financiamiento: conocimiento o desconocimiento por las pymes de Saltillo. Políticas Sociales Sectoriales2.

Deci, E. L., \& Ryan, R. M. (2000). The "what" and "why" of goal pursuits: Human needs and the self-determination of behavior. Psychological Inquiry, 11(4), 227-268. https://doi.org/10.1207/S15327965PLI1104_01

Devotto, R. P., \& Wechsler, S. M. (2019). Job Crafting Interventions: Systematic Review. Temas Em Psicologia, 27(2), 371-383. https://doi.org/10.9788/tp2019.2-06

Escamilla Salazar, Z., Caldera González, D., \& Cruz del Castillo, C. (2014). El emprendedor potencial: identificación de oportunidades relacionadas con algunas variables del capital humano y social. Entreciencias, 2(5), 245-26.

Freeman, R. B. (1978). Job satisfaction as an economic variable. American Economic Review, 68(2), 135-141. Retrieved from http://www.jstor.org/stable/1816677

Freire Seoane, M. J., \& Teijeiro Álvarez, M. (2009). Análisis de los factores que afectan a la decisión de ser emprendedor. Cuadernos de Economía, 32(90), 5-27. https://doi.org/10.1016/s0210-0266(09)70052-4

Han, B. (2014). Psicopolítica. Herder.

Hernández García, C., \& Tomé González, A. (2016). Prcepción acerca del bienestar : las diferencias por género en México. XXI Congreso Internacional de Contaduría, Administración e Informática, 1-19.

Instituto Nacional de Estadística y Geografía. (2014). Bienestar subjetivo. BIARE. Ampliado. Módulo de Bienestar Autorreportado, Módulo de Condiciones Socioeconómicas 2014, 2015, 2016. MCS. Retrieved from http:// www.beta.inegi.org.mx/proyectos/investigacion/bienestar/ampliado/

Kautonen, T., Kibler, E., \& Minniti, M. (2017). Late-career entrepreneurship, income and quality of life. Journal of Business Venturing, 32(3), 318-333. https://doi.org/10.1016/j.jbusvent.2017.02.005

Koellinger, P., Minniti, M., \& Schade, C. (2007). "I think I can, I think I can": Overconfidence and entrepreneurial behavior. Journal of Economic Psychology, 28(4), 502-527. https://doi.org/10.1016/j.joep.2006.11.002

Moriano León, J. A., Palací Descals, F. J., \& Morales Domínguez, J. F. (2006). El perfil psicosocial del emprendedor universitario. Revista de Psicología Del Trabajo y La Organizaciones, 22(1), 75-99.

Muñiz, C., \& Ramírez, J. (2015). Tratamiento informativo de las reacciones empresariales ante situaciones de violencia e inseguridad. Estudios Sobre El Mensaje Periodístico, 21(1), 437-453.

Naudé, W., Amorós, J. E., \& Cristi, O. (2014). "Surfeiting, the appetite may sicken”: Entrepreneurship and happiness. Small Business Economics, 42(3), 523-540. https://doi.org/10.1007/s11187-013-9492-x

Ohtake, F. (2012). Unemployment and Happiness. Japan Labor Review, 9(2), 59-74.

Patel, P. C., Wolfe, M. T., \& Williams, T. A. (2019). Self-employment and allostatic load. Journal of Business Venturing, 34(4), 731-751. https://doi.org/10.1016/j.jbusvent.2018.05.004

Raccanello, K., \& Saucedo Carranza, C. B. (2015). Microempresas y microcréditos en la ciudad de Puebla. Perspectivas. Revista de Análisis de Economía, Comercio y Negocios Internacionales, 9(1), 5-27. 
Ríos, V. (2012). Security issues and immigration flows: Drug-violence refugees, the new Mexican immigrants. Latin American Research Review, 2011, 1-31. Retrieved from http://www.gov.harvard.edu/files/RiosV2014_ LARR2014_SecurityImmigration.pdf

Rojas, M. (2007). Estatus económico y situación afectiva en América Latina. Estudos Contemporaneos Da Subjetividade, 3(2), 202-218.

Rojas, M. (2015). Suffering Ailments and Addiction Problems in the Family. https://doi.org/10.1007/978-94-0179670-5

Shane, S., \& Venkataraman, S. (2000). The Promise of Enterpreneurship as a Field of Research. The Academy of Management Review, 25(1), 217-226. https://doi.org/10.2307/259271

Sherman, C. L., Randall, C., \& Kauanui, S. K. (2016). Are you happy yet? Entrepreneurs' subjective well-being. Journal of Management, Spirituality and Religion, 13(1), 7-23. https://doi.org/10.1080/14766086.2015.1043 575

Silva, J. C. da, Dal Magro, C. B., Gorla, M. C., \& Silva, M. Z. da. (2018). Glass ceiling in the accounting profession: Evidence in Brazilian companies. Contaduría y Administración, 63(2), 16. https://doi.org/10.22201/ fca.24488410e.2018.928

Temkin, B. (2016). The Negative Influence of Labour Informality in Subjective Well-Being. Global Labour Journal, 7(1), 69-93. https://doi.org/10.15173/glj.v7i1.2545

Varela Llamas, R., Ocegueda Hernández, J. M., Castillo Ponce, R. A., \& Huber Bernal, G. (2016). Determinantes de los ingresos salariales en México: una perspectiva de capital humano. Región Y Sociedad, 22(49). https:// doi.org/10.22198/rys.2010.49.a424

Villagómez, F. A. (2014). El ahorro para el retiro: Una reflexión para México. Trimestre Economico, 81(323), 549-576. https://doi.org/10.20430/ete.v81i323.122

Wiklund, J., Nikolaev, B., Shir, N., Foo, M. Der, \& Bradley, S. (2019). Entrepreneurship and well-being: Past, present, and future. Journal of Business Venturing, 34(4), 579-588. https://doi.org/10.1016/j.jbusvent.2019.01.002

Winkelmann, L., \& Winkelmann, R. (1998). Why Are the Unemployed So Unhappy? Evidence from Panel Data. Economica, 65, 1-15.

Yamada, G., \& Oviedo, N. (2016). Educación superior y subempleo profesional: ¿ una creciente burbuja mundial? Yepes-Baldó, M., Romeo, M., Westerberg, K., \& Nordin, M. (2018). Job Crafting, Employee Well-being, and Quality of Care. Western Journal of Nursing Research, 40(1), 52-66. https://doi.org/10.1177/0193945916680614 